\title{
Applying Variable Precision Rough Set for Clustering Diabetics Dataset
}

\author{
Tutut Herawan ${ }^{1}$ and Wan Maseri Wan Mohd ${ }^{2}$ \\ ${ }^{1}$ Department of Mathematics Education, Universitas Ahmad Dahlan \\ Jalan Prof Dr Soepomo 55166, Yogyakarta, Indonesia \\ ${ }^{2}$ Faculty of Computer System and Software Engineering \\ Universiti Malaysia Pahang \\ Lebuh Raya Tun Razak, Gambang 26300, Kuantan, Pahang, Malaysid \\ tutut81@uad.ac.id,maseri@ump.édusmy \\ Abstract
}

Computational models of the artificial intelligence such as rough set theory have several applications. Rough set-based data clustering can be considered further as a technique for medical decision making. This paper presents the results of an experimental study of a roughset based clustering technique using Variable Precision Rough Set (VPRS). Here, we employ our proposed clustering technique [12f through a medical dataset of patients suspected diabetic. Our results indicate that the VPRS-based technique is better than that the standard rough set-based techniques in the process of selecting a clustering attribute.

Keywords: Clustering; Rough set; Variable precision rough set model; Diabetic dataset

\section{Introduction}

Clustering is a division of dat into groups of similar objects. Each group, called a cluster, consists of objects that are similar to one another and dissimilar to objects of other groups. When representing data with fewer clusters necessarily loses certain fine details (akin to lossy data compression), butachieves simplification [1]. We refer the reader to [1,2] for a review of cluster analysis. Several cluster analysis techniques have been developed so far to group objects having similar characteristics. Clustering of categorical data is more challenging than that of numerical data. Most of the early cluster analysis techniques face problems due to the fact that mach of the data contained in today's databases is categorical in nature. This necessitated the development of some algorithms for clustering categorical data [3]. For categotical data clustering, a new trend has become in algorithms which can handle uncertainty in the clustering process. One of the well-known techniques is based on rough set theory [4-6]. The main idea of the rough clustering is the clustering data set is mapped as the decision table. This can be done by introducing a decision attribute. Further, a divide-andconquer method can be used to partition/cluster the objects. One of the successful pioneering rough clustering for categorical data techniques is Total Roughness [7] and MinimumMinimum Roughness (MMR) [8]. These algorithms are based on the notions of lower and upper approximations of a set. Later a rough set-based attributes dependency technique is proposed for handling the problem of computational complexity of TR and MMR, namely MDA [9-10]. However, in reviewing the above techniques, they are developed based on standard rough set model. To this, in facing noisy datasets as an integral part of databases, they fail to do. In order to overcome their drawback, an error parameter is introduced. 
Variable Precision Rough Set (VPRS) model proposed by Ziarko [11] is defined on the probabilistic space and will give us a new way to deal with the noisy data. In previous work, we have proposed an alternative technique for clustering noisy categorical data using Variable Precision Rough Set model [12-13]. It is shown that the proposed technique performs better than that the TR, MMR and MDA in handling noisy datasets.

Inspired by VPRS for handling noisy data, in this paper, we present the results of an experimental study of a rough-set based clustering technique using VPRS. Here, we employ our proposed clustering technique [11] through a medical survey dataset of patients suspected diabetic. Our results indicate that the VPRS-based technique is better than that the standard rough set-based techniques [7-10] in the process of selecting a clustering attribute.

The rest of this paper is organized as follows. Section 2 describes the notion of rough set theory which comprises an information system, set approximations and yariable precision rough set model. Section 3 presents a short description on rough set-based techniques for selecting a clustering attribute, following by the proposed VPRS technique. Section 4 describes the experimental tests. Finally, the conclusion of this work is described in Section 5.

\section{Variable Precision Rough Set}

\subsection{Set Approximations}

Motivation for rough set theory has come from the need to represent subsets of a universe in terms of equivalence classes of a partition of the unverse [14,15]. Here, we use the concept of rough set theory in term of dat containing in an information system. An information system as in [5] is a 4-tuple (quadruple) $S_{0}=(U, A, V, f)$, where $U$ is a non-empty finite set of objects, $A$ is a non-empty finjte set of attributes, $V=\bigcup_{a \in A} V_{a}, V_{a}$ is the domain (value set) of attribute $a, f: U \times A \ngtr V$ is a total function such that $f(u, a) \in V_{a}$, for every $(u, a) \in U \times A$, called information (nnowledge) function.

Definition 1. Let $S=(U, A, R)$ be an information system and let $B$ be any subset of $A$. Two elements $x, y \in U$ is said tobe $B$-indiscernible (indiscernible by the set of attribute $B \subseteq A$ in $S)$ if and only if $f(x, a)=f(y, a)$, for every $a \in B$.

Obviously, évery subset of $A$ induces unique indiscernibility relation. Notice that, an indiscernibility relation induced by the set of attribute $B$, denoted by $\operatorname{IND}(B)$, is an equivalence relation. It is well known that, an equivalence relation induces unique partition. The partition of $U$ induced by $\operatorname{IND}(B)$ in $S=(U, A, V, f)$ denoted by $U / B$ and the equivalence class in the partition $U / B$ containing $x \in U$, denoted by $[x]_{B}$.

The notions of lower and upper approximations of a set can be defined as follows:

Definition 2. Let $S=(U, A, V, f)$ be an information system, let $B$ be any subset of $A$ and let $X$ be any subset of $U$. The B-lower approximation of $X$, denoted by $\underline{B}(X)$ and $B$-upper approximations, denoted by $\bar{B}(X)$ of $X$, respectively, are defined by

$\underline{B}(X)=\left\{x \in U \mid[x]_{B} \subseteq X\right\}$ and $\bar{B}(X)=\left\{x \in U \mid[x]_{B} \cap X \neq \phi\right\}$. 


\subsection{Variable Precision Rough Set}

Variable precision rough set (VPRS) extends standard rough set theory by the relaxation of the subset operator [11]. It was proposed to analyze and identify data patterns which represent statistical trends rather than functional. The main idea of VPRS is to allow objects to be classified with an error smaller than a certain pre-defined level. This introduced threshold relaxes the rough set notion of requiring no information outside the dataset itself.

Definition 3. Let a set $U$ as a universe and $X, Y \subseteq U$, where $X, Y \neq \phi$. The error classification rate of $X$ relative to $Y$ is denoted by $e(X, Y)$, is defined by

$$
e(X, Y)=\left\{\begin{array}{ll}
1-\frac{|X \cap Y|}{|X|}, & |X|>0 \\
0 & ,|X|=0
\end{array} .\right.
$$

Definiton 4. Let $U$ be a finite set and let $\beta$ be a real number within the range $0 \leq \beta<0.5$ and a set $X \subseteq U$. The $B_{\beta}$-lower approximation of $X$, denoted by $\underline{B}_{\beta}(X)$ and $B_{\beta}$-upper approximation of $X$, denoted by $\bar{B}_{\beta}(X)$, respectively, are defined by

$$
\underline{B}_{\beta}(X)=\left\{x \in U: e\left([x]_{B}, X\right) \leq \beta\right\} \text { and } \bar{B}(X)=\left\{x \in \mathcal{U} \cdot e\left([x]_{B}, X\right)<1-\beta\right\} .
$$

The set $\underline{B}_{\beta}(X)$ is called the positive region $\theta$ f $X$. It's the set of object of $U$ that can be classified into $X$ with error classification rate not greater than $\beta$. Then we have $\underline{B}_{\beta}(X) \subseteq \bar{B}_{\beta}(X)$ if only if $0 \leq \beta \leq\{0.5$, which means that $\beta$ be restricted in an interval $[0,0.5)$ in ordep to keep the meaning of the "upper" and "lower" approximations.

\section{Rough Set-based Techniques for Selecting a Clustering Attribute}

In our previous work [12], we analyze two establish rough-set based techniques for selecting a clustering attribute. The first technique called Total Roughness (TR) is proposed by Mazlack et al.,' [7]. With the TR technique, the attribute selected as the clustering (partitioning) attribute is based on the maximum value of mean roughness. The second techniquercalled Min-Min Roughness (MMR) is proposed by Parmar et al., [8]. The least of mean roughness of an attribute is the best attribute to be selected. Later, in [9,10], we showed that in almost all type of non-noisy database, the techniques of TR, MMR and MDA have the same result in selecting a clustering attribute. However, for noisy datasets they fail to handle, since no recommendation for selection a clustering attribute.

In this section, we present the proposed technique, which we refer to as Maximum Accuracy of Variable Precision Rough Set [12]. The technique uses the accuracy of approximation using variable precision of attributes in the rough set theory. The higher accuracy of approximation using variable precision of attributes is the more accurate (higher of accuracy of stranded approximation) for selecting clustering attribute. The justification that the accuracy of approximation using variable precision of attributes, the more accurate for selecting clustering attribute is stated in Proposition 1. 
Definition 5. The accuracy of approximation variable precision (accuracy of variable precision roughness) of any subset $X \subseteq U$ with respect to $B \subseteq A$ is denoted by $\alpha_{B_{\beta}}(X)$. It presented as

$\alpha_{B_{\beta}}(X)=\frac{\left|\underline{B}_{\beta}(X)\right|}{\left|\bar{B}_{\beta}(X)\right|}$

where $|X|$ denotes cardinality of $X$. If $\beta=0$, it is the traditional rough set model of Pawlak.

Proposition 1. Let $S=(U, A, V, f)$ be an information system, $\alpha_{B}(X)$ be an accuracy of roughness, $\alpha_{B_{\beta}}(X)$ is an accuracy of variable precision roughness and given $\beta$ the error factor of variable precision. If $(0 \leq \beta<0.5)$, then $\alpha_{B}(X) \leq \alpha_{B}(X)$.

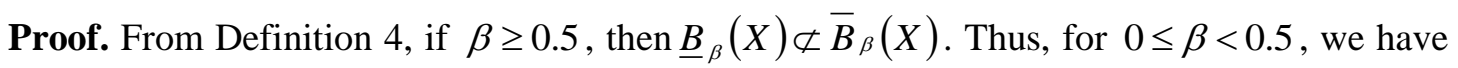
$\underline{B}_{0}(X) \supseteq \underline{B}_{\beta}(X) \quad$ and $\quad \bar{B}_{0}(X) \subseteq \bar{B}_{\beta}(X)$. Consequently, $\quad\left|\underline{B}_{0}(X)\right| \leq\left|\underline{B}_{\beta}(X)\right| \quad$ and $\left|\bar{B}_{0}(X)\right| \geq\left|\bar{B}_{\beta}(X)\right|$. There are two cases for the yalue of $\beta$ Fe for $\beta=0$, from Definition 4 , we have $\alpha_{B}(X)=\alpha_{B_{\beta}}(X)$. While, for $0<\beta<0.5$, we baye

$|\underline{B}(X)| \leq\left|\underline{B}_{\beta}(X)\right|$ and $\left|\bar{B}_{\beta}(X)\right| \leq \mid \bar{B}(X)$

Hence

$$
\frac{|\underline{B}(X)|}{|\bar{B}(X)|} \leq \frac{\left|\underline{B}_{\beta}(X)\right|}{\left|\bar{B}_{\beta}(X)\right|} .
$$

Therefore, $\alpha_{B}(X) \leqslant \alpha_{B_{\beta}}(X)$.

Definition 6. Suppose $a_{i} \in A, V\left(a_{i}\right)$ has $k$-different values, say $\gamma_{k}, k=1,2, \cdots, n$. Let $X\left(a_{i}=\gamma_{k}\right), k=1,2, \cdots$ be a subset of the objects having $k$-different values of attribute $a_{i}$. The accuracy of the set $X\left(a_{i}=\gamma_{k}\right), k=1,2, \cdots, n$ for given $\beta$ error factor, with respect to $a_{j}$, where $i$ j, denoted $\alpha_{\beta a_{j}}\left(X \mid a_{i}=\gamma_{k}\right)$, is defined by

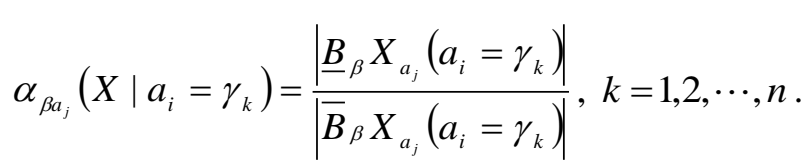

The mean accuracy of attribute $a_{i} \in A$ with respect to $a_{j} \in A$, where $i \neq j$, denoted by $\operatorname{MAC}_{a_{j}}\left(a_{i}\right)$, is evaluated as follows

$$
\operatorname{MAC}_{a_{j}}\left(a_{i}\right)=\frac{\sum_{k=1}^{\left|V\left(a_{i}\right)\right|} \alpha_{\beta a_{j}}\left(X \mid a_{i}=\gamma_{k}\right)}{\left|V\left(a_{i}\right)\right|}
$$

where $V\left(a_{i}\right)$ is the set of values of attribute $a_{i} \in A$. 
Definition 7. Given $n$ attributes, maximal accuracy of attribute $a_{i} \in A$ with respect to $a_{j} \in A$, where $i \neq j$, refers to the maximum of $\operatorname{MAC}_{a_{j}}\left(a_{i}\right)$, denoted $\operatorname{MA}\left(a_{i}\right)$, is obtained by the following formula

$$
\operatorname{MA}\left(a_{i}\right)=\operatorname{mean}\left(\operatorname{MAC}_{a_{j}}\left(a_{i}\right)\right), \quad 1 \leq i, j \leq n
$$

Figure 1 shows the pseudo-code of the proposed technique.

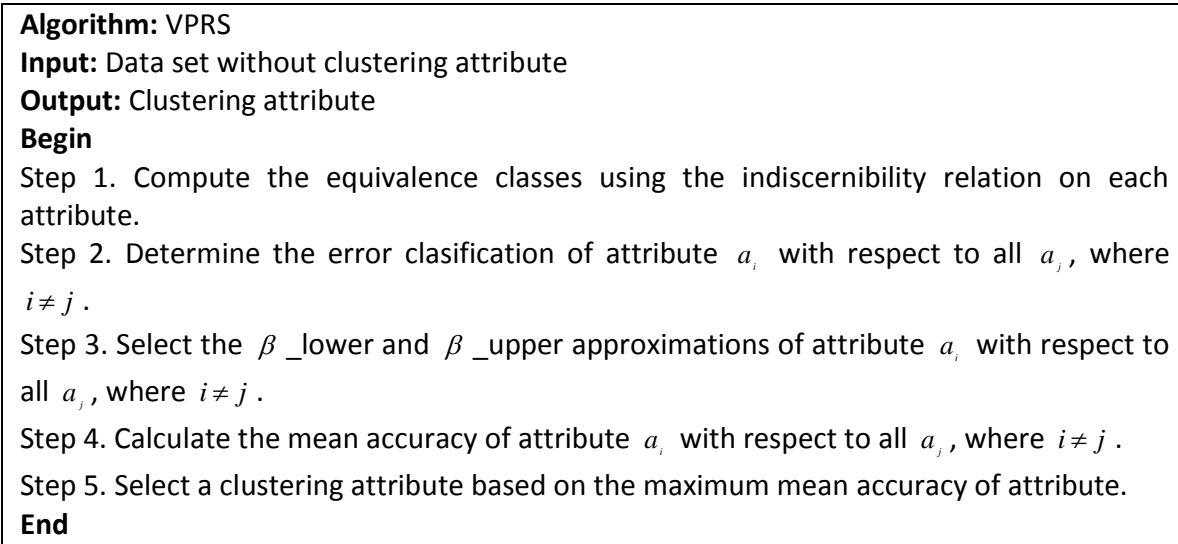

Figure 1. The pseudo-code of VPRSfor selecting a clustering attribute

\section{Experiment Results}

\subsection{Material}

Diabetes mellitus is nor a major global public health problem. The incidence and prevalence of diabetes are escalating especially developing and newly industrialized nations. The estimated number of 80 million sufferers in 1990 is expected to double by the year 2000 . In Asia alone, it is estimated that the total number of diabetes could reach more than 138 million [16]. Ln Malaysia, hundreds of thousands of people are afflicted with this chronic disease. In thi work, the data of patients suspected diabetic was collected from a survey in Kuantan Pahang Malaysia. There are 252 suspects of diabetic and ten symptoms founded; Often Thirst, Excessive Hunger, Frequent Urination, Tiredness and Fatigue, Rapid and/or Sudden Weight Loss, Blurred Vision, Numbness and/or Tingling in the Hands and Feet, Slow healing of Minor-to-treat Yeast Infection in Women, Recurrent or Hard-o-treat Yeast Infection in Women, Dry or Itchy Skin. All attributes has two values (yes/no), signifying the absence or presence of some feature. If the attribute value is yes, then the symptom is occur and otherwise is not. The summary dataset is summarized in Table 1. 
Table 1. A summary of the diabetic dataset

\subsection{Clustering problem}

\begin{tabular}{|l|l|}
\hline Symptoms & Number of suspects \\
\hline Often Thirst (OT) & 81 \\
\hline Excessive Hunger (EH) & 81 \\
\hline Frequent Urination (FE) & 81 \\
\hline Tiredness and Fatigue (TF) & 81 \\
\hline Rapid and/or Sudden Weight Loss (WL) & 81 \\
\hline Blurred Vision (BV) & 45 \\
\hline $\begin{array}{l}\text { Numbness and/or Tingling in the Hands } \\
\text { and Feet (THF) }\end{array}$ & 45 \\
\hline $\begin{array}{l}\text { Slow healing of Minor-to-treat Yeast } \\
\text { Infection in Women (SYI) }\end{array}$ & 45 \\
\hline $\begin{array}{l}\text { Recurrent or Hard-o-treat Yeast Infection } \\
\text { in Women (RYI) }\end{array}$ & 45 \\
\hline Dry or Itchy Skin (IS) & 43 \\
\hline
\end{tabular}

The problem of the VPRS-based data clustering trough the above dataset is how to clusters the suspects of diabetic in the same group with similar characteristics (symptoms). It can be done, firstly among all attributes we want to select a clustering attribute. To select the candidate, we employ the proposed technique described in sub-section 3. Lastly, the divide and conquer technique is used to group the guspects haring the same symptoms.

\subsection{Result}

In this experiment we choose the value of $\beta \approx 0.4$. The algorithm of TR, MMR, MDA and VPRS are implemented in MATLAB version 7.6.0.324 (R2008a). They are executed sequentially on a processor Intel Core 2 Duo CPUs. The total main memory is $4 \mathrm{G}$ and the operating system is Windows XP Professional SP3. The results are described in Tables 2, 3, 4 and 5 , respectiyely

Tabie 2. The mean roughness value using TR technique

\begin{tabular}{|c|c|c|c|c|c|c|c|c|c|c|}
\hline Att & \multicolumn{9}{|c|}{ Mean Roughness } & \multirow{2}{*}{$\begin{array}{l}\text { Mean } \\
0\end{array}$} \\
\hline \multirow[t]{2}{*}{ OT } & $\mathrm{EH}$ & $F E$ & $\mathrm{TF}$ & WL & BV & THF & SYI & RYI & IS & \\
\hline & 0 & 0 & 0 & 0 & 0 & 0 & 0 & 0 & 0 & \multirow{3}{*}{0} \\
\hline Ef & $\mathrm{OT}^{\prime}$ & FE & $\mathrm{TF}$ & WL & BV & THF & SYI & RYI & IS & \\
\hline & $\theta$ & 0 & 0 & 0 & 0 & 0 & 0 & 0 & 0 & \\
\hline & OT & $\mathrm{EH}$ & $\mathrm{TF}$ & WL & BV & THF & SYI & RYI & IS & \multirow[t]{2}{*}{0} \\
\hline & 0 & 0 & 0 & 0 & 0 & 0 & 0 & 0 & 0 & \\
\hline \multirow[t]{2}{*}{$\mathrm{TF}$} & OT & $\mathrm{EH}$ & FE & WL & $\mathrm{BV}$ & THF & SYI & RYI & IS & \multirow[t]{2}{*}{0} \\
\hline & 0 & 0 & 0 & 0 & 0 & 0 & 0 & 0 & 0 & \\
\hline \multirow[t]{2}{*}{$\mathrm{WL}$} & OT & $\mathrm{EH}$ & FE & $\mathrm{TF}$ & $\mathrm{BV}$ & THF & SYI & RYI & IS & \multirow[t]{2}{*}{0} \\
\hline & 0 & 0 & 0 & 0 & 0 & 0 & 0 & 0 & 0 & \\
\hline \multirow[t]{2}{*}{$\mathrm{BV}$} & OT & $\mathrm{EH}$ & FE & $\mathrm{TF}$ & WL & THF & SYI & RYI & IS & \multirow[t]{2}{*}{0} \\
\hline & 0 & $\begin{array}{l}0 \\
\end{array}$ & 0 & 0 & 0 & $\begin{array}{l}0 \\
\end{array}$ & 0 & $\begin{array}{l}0 \\
\end{array}$ & 0 & \\
\hline \multirow[t]{2}{*}{ THF } & OT & $\mathrm{EH}$ & FE & TF & WL & BV & SYI & RYI & IS & \multirow[t]{2}{*}{0} \\
\hline & 0 & 0 & 0 & 0 & 0 & 0 & 0 & 0 & 0 & \\
\hline \multirow[t]{2}{*}{ SYI } & OT & $\mathrm{EH}$ & $\mathrm{FE}$ & $\mathrm{TF}$ & WL & $\mathrm{BV}$ & THF & RYI & IS & \multirow[t]{2}{*}{0} \\
\hline & 0 & 0 & 0 & 0 & 0 & 0 & 0 & 0 & 0 & \\
\hline \multirow[t]{2}{*}{ RYI } & OT & $\mathrm{EH}$ & FE & TF & WL & $\mathrm{BV}$ & THF & SYI & IS & \multirow[t]{2}{*}{0} \\
\hline & 0 & 0 & 0 & 0 & 0 & 0 & 0 & 0 & 0 & \\
\hline \multirow[t]{2}{*}{ IS } & OT & $\mathrm{EH}$ & FE & $\mathrm{TF}$ & WL & BV & THF & SYI & RYI & \multirow[t]{2}{*}{0} \\
\hline & 0 & 0 & 0 & 0 & 0 & 0 & 0 & 0 & 0 & \\
\hline
\end{tabular}


Table 3. The mean roughness using of MMR technique

\begin{tabular}{|c|c|c|c|c|c|c|c|c|c|c|}
\hline \multirow{3}{*}{$\frac{\text { Att }}{\text { OT }}$} & \multicolumn{9}{|c|}{ Mean Roughness } & \multirow{2}{*}{$\begin{array}{l}\text { Mean } \\
1\end{array}$} \\
\hline & $\mathrm{EH}$ & $\mathrm{FE}$ & TF & WL & $\mathrm{BV}$ & THF & SYI & RYI & IS & \\
\hline & 1 & 1 & 1 & 1 & 1 & 1 & 1 & 1 & 1 & \\
\hline \multirow[t]{2}{*}{$\mathrm{EH}$} & OT & $\mathrm{FE}$ & $\mathrm{TF}$ & WL & $\mathrm{BV}$ & THF & SYI & RYI & IS & \multirow[t]{2}{*}{1} \\
\hline & 1 & 1 & 1 & 1 & 1 & 1 & 1 & 1 & 1 & \\
\hline \multirow[t]{2}{*}{$\mathrm{FE}$} & OT & $\mathrm{EH}$ & TF & WL & $\mathrm{BV}$ & THF & SYI & RYI & IS & \multirow[t]{2}{*}{1} \\
\hline & 1 & 1 & 1 & 1 & 1 & 1 & 1 & 1 & 1 & \\
\hline \multirow[t]{2}{*}{$\mathrm{TF}$} & OT & $\mathrm{EH}$ & $\mathrm{FE}$ & WL & $\mathrm{BV}$ & THF & SYI & RYI & IS & \multirow[t]{2}{*}{1} \\
\hline & 1 & 1 & 1 & 1 & 1 & 1 & 1 & 1 & 1 & \\
\hline \multirow[t]{2}{*}{ WL } & OT & $\mathrm{EH}$ & FE & TF & $\mathrm{BV}$ & THF & SYI & RYI & IS & \multirow{4}{*}{${ }^{1}$} \\
\hline & 1 & 1 & 1 & 1 & 1 & 1 & 1 & 1 & 1 & \\
\hline \multirow[t]{2}{*}{$\mathrm{BV}$} & OT & $\mathrm{EH}$ & FE & TF & WL & THF & SYI & RYI & IS & \\
\hline & 1 & 1 & 1 & 1 & 1 & 1 & 1 & 1 & $1 /$ & \\
\hline \multirow[t]{2}{*}{ THF } & OT & $\mathrm{EH}$ & FE & TF & WL & BV & & RYI & 15 & \multirow{2}{*}{1} \\
\hline & 1 & 1 & 1 & 1 & 1 & 1 & & 12 & 1 & \\
\hline \multirow[t]{2}{*}{ SYI } & OT & $\mathrm{EH}$ & FE & TF & WL & BV & THF & RYI & IS & \multirow{2}{*}{1} \\
\hline & 1 & 1 & 1 & 1 & 1 & 1 & 1 & & 1 & \\
\hline \multirow[t]{2}{*}{ RYI } & OT & $\mathrm{EH}$ & FE & TF & WL & $\mathrm{BV}$ & THF & SYI & IS & \multirow[t]{2}{*}{1} \\
\hline & 1 & 1 & 1 & 1 & 1 & $y$ & 1 & 1 & 1 & \\
\hline \multirow[t]{2}{*}{ IS } & OT & $\mathrm{EH}$ & FE & TF & $\mathrm{WL}$ & BV & $\mathrm{THF}$ & SYI & RYI & \multirow[t]{2}{*}{1} \\
\hline & 1 & 1 & 1 & 1 & & 1 & 17 & 1 & 1 & \\
\hline
\end{tabular}

Table 4. The dependency degree using MDA technique

\begin{tabular}{|c|c|c|c|c|c|c|c|c|c|c|}
\hline \multirow{3}{*}{$\frac{\text { Att }}{\text { OT }}$} & \multicolumn{9}{|c|}{ Dependency } & \multirow{3}{*}{$\begin{array}{l}\text { MDA } \\
0\end{array}$} \\
\hline & \multicolumn{5}{|c|}{$\mathrm{EH} \quad \mathrm{FE}_{\mathrm{TF}}$} & \multirow{2}{*}{$\begin{array}{l}\text { THF } \\
0\end{array}$} & \multirow{2}{*}{$\begin{array}{l}\text { SYI } \\
0\end{array}$} & \multirow{2}{*}{$\begin{array}{l}\text { RYI } \\
0\end{array}$} & \multirow{2}{*}{$\begin{array}{l}\text { IS } \\
0\end{array}$} & \\
\hline & 0 & 0 & 0 & 0 & 0 & & & & & \\
\hline \multirow[t]{2}{*}{$\mathrm{EH}$} & $\overline{\text { OT }}$ & $\overrightarrow{\mathrm{FE}}$ & $\mathrm{TF}$ & WL & $\mathrm{BV}$ & THF & SYI & RYI & IS & \multirow[t]{2}{*}{0} \\
\hline & 0 & $\sigma$ & A & 8 & 0 & 0 & 0 & 0 & 0 & \\
\hline \multirow[t]{2}{*}{$\mathrm{FE}$} & OT & $\mathrm{EH}$ & $\mathrm{TF}$ & WL & BV & THF & SYI & RYI & IS & \multirow[t]{2}{*}{0} \\
\hline & 0 & 0 & & 0 & 0 & 0 & 0 & 0 & 0 & \\
\hline \multirow[t]{2}{*}{$\mathrm{TF}$} & OT & EH & $\mathrm{FE}$ & WL & $\mathrm{BV}$ & THF & SYI & RYI & IS & \multirow[t]{2}{*}{0} \\
\hline & 0 & $a=$ & 0 & 0 & 0 & 0 & 0 & 0 & 0 & \\
\hline \multirow[t]{2}{*}{ WL } & OT & $\mathrm{EH}$ & FE & TF & $\mathrm{BV}$ & THF & SYI & RYI & IS & \multirow[t]{2}{*}{0} \\
\hline & 0 & 0 & 0 & 0 & 0 & 0 & 0 & 0 & 0 & \\
\hline \multirow[t]{2}{*}{$\mathrm{BV}$} & OTY & $\mathrm{EH}$ & FE & TF & WL & THF & SYI & RYI & IS & \multirow[t]{2}{*}{0} \\
\hline & 0 & 0 & 0 & 0 & 0 & 0 & 0 & 0 & 0 & \\
\hline & OT & $\mathrm{EH}$ & FE & $\mathrm{TF}$ & WL & BV & SYI & RYI & IS & \multirow[t]{2}{*}{0} \\
\hline & 0 & 0 & 0 & 0 & 0 & 0 & 0 & 0 & 0 & \\
\hline \multirow[t]{2}{*}{ SYI } & OT & $\mathrm{EH}$ & FE & TF & WL & $\mathrm{BV}$ & THF & RYI & IS & \multirow[t]{2}{*}{0} \\
\hline & 0 & 0 & 0 & 0 & 0 & 0 & 0 & 0 & 0 & \\
\hline \multirow[t]{2}{*}{ RYI } & OT & EH & FE & $\mathrm{TF}$ & WL & BV & THF & SYI & IS & \multirow[t]{2}{*}{0} \\
\hline & 0 & 0 & 0 & 0 & 0 & 0 & 0 & 0 & 0 & \\
\hline \multirow[t]{2}{*}{ IS } & OT & $\mathrm{EH}$ & FE & TF & WL & $\mathrm{BV}$ & THF & SYI & RYI & \multirow[t]{2}{*}{0} \\
\hline & 0 & 0 & 0 & 0 & 0 & 0 & 0 & 0 & 0 & \\
\hline
\end{tabular}


Table 5. The mean roughness using of VPRS technique

\begin{tabular}{|c|c|c|c|c|c|c|c|c|c|c|}
\hline \multirow{3}{*}{$\begin{array}{c}\text { Att } \\
\text { OT }\end{array}$} & \multicolumn{9}{|c|}{ Mean Roughness } & \multirow{2}{*}{$\begin{array}{l}\text { Mean } \\
0\end{array}$} \\
\hline & EH & $\mathrm{FE}$ & $\mathrm{TF}$ & WL & $\mathrm{BV}$ & THF & SYI & RYI & IS & \\
\hline & 0 & 0 & 0 & 0 & 0 & 0 & 0 & 0 & 0 & \\
\hline \multirow[t]{2}{*}{$\mathrm{EH}$} & OT & FE & $\mathrm{TF}$ & WL & $\mathrm{BV}$ & THF & SYI & RYI & IS & \multirow[t]{2}{*}{0} \\
\hline & 0 & 0 & 0 & 0 & 0 & 0 & 0 & 0 & 0 & \\
\hline \multirow[t]{2}{*}{ FE } & OT & EH & $\mathrm{TF}$ & WL & BV & THF & SYI & RYI & IS & \multirow[t]{2}{*}{0} \\
\hline & 0 & 0 & 0 & 0 & 0 & 0 & 0 & 0 & 0 & \\
\hline \multirow[t]{2}{*}{$\mathrm{TF}$} & OT & EH & $\mathrm{FE}$ & WL & BV & THF & SYI & RYI & IS & \multirow[t]{2}{*}{0} \\
\hline & 0 & 0 & 0 & 0 & 0 & 0 & 0 & 0 & 0 & \\
\hline \multirow[t]{2}{*}{ WL } & OT & EH & FE & $\mathrm{TF}$ & $\mathrm{BV}$ & THF & SYI & RYI & IS & \multirow[b]{4}{*}{$\Delta y$} \\
\hline & 0 & 0 & 0 & 0 & 0 & 0 & 0 & 0 & 0 & \\
\hline \multirow[t]{2}{*}{$\mathrm{BV}$} & OT & EH & $\mathrm{FE}$ & $\mathrm{TF}$ & WL & THF & SYI & RYI & IS & \\
\hline & 0 & 0 & 0 & 0 & 0 & 0 & 0 & 0 & 0 & \\
\hline \multirow[t]{2}{*}{ THF } & OT & EH & FE & $\mathrm{TF}$ & $\mathrm{WL}$ & $\mathrm{BV}$ & SYI & RYI & 15 & \multirow{2}{*}{$\gamma^{\prime}$} \\
\hline & 0 & 0 & 0 & 0 & 0 & 0 & 0 & 0 & 0 & \\
\hline \multirow[t]{2}{*}{ SYI } & OT & EH & FE & $\mathrm{TF}$ & WL & $\mathrm{BV}$ & TH & RYA & IS & \multirow[t]{2}{*}{0} \\
\hline & 0 & 0 & 0 & 0 & 0 & 6 & $\vec{\theta}$ & 0 & $\varnothing$ & \\
\hline \multirow[t]{2}{*}{ RYI } & OT & EH & FE & $\mathrm{TF}$ & WL & $\mathrm{BV}$ & THF & SYI & IS & \multirow[t]{2}{*}{0} \\
\hline & 0 & 0 & 0 & 0 & 0 & 0 & 0 & 0 & 0 & \\
\hline \multirow[t]{2}{*}{ IS } & OT & EH & FE & $\mathrm{TF}$ & WL & $B V$ & THF & SYI & RYI & \multirow{2}{*}{0.0276} \\
\hline & 0 & 0 & 0 & $0.2480^{\circ}$ & & 0 & 0 & 0 & 0 & \\
\hline
\end{tabular}

From Table 5, the selected attributes is Dry or ltchy Skin (IS). Therefore we have two initial clusters of patients $(\mathrm{P})$, i.e.. 1

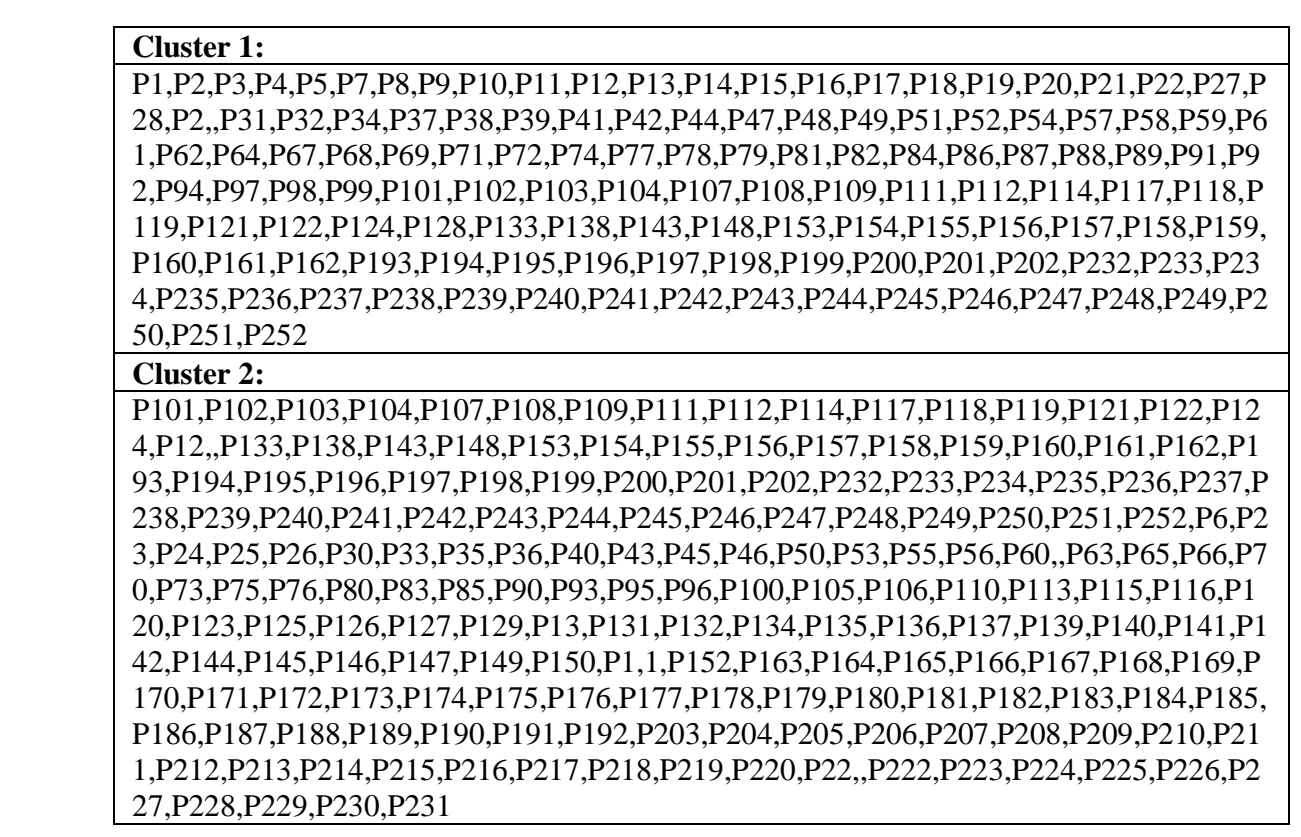

Figure 1. The clusters obtained

From Figure 1, the divided and conquer technique can be applied recursively to obtain further clusters. At subsequent iterations, the leaf node having more objects is selected for further splitting. The splitting processes terminate when it reaches a pre-defined number of clusters. This is subjective and is pre-decided based either on user requirement or domain knowledge. 


\subsection{Cluster purity and its visualization}

The purity of clusters was used as a measure to test the quality of the clusters [5]. The purity of a cluster and overall purity are defined as

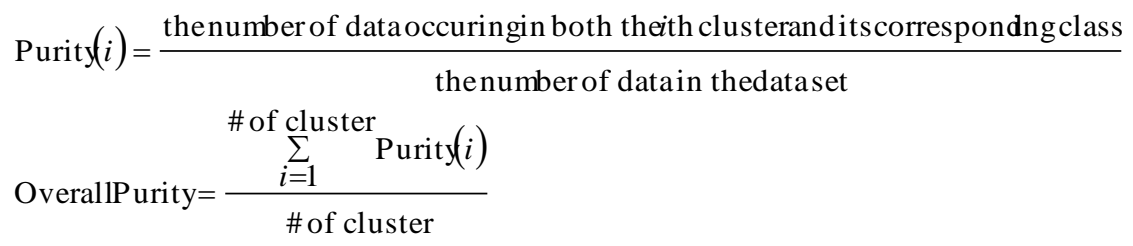

According to this measure, a higher value of overall purity indicates a better clustering result, with perfect clustering yielding a value of 1 [5].

For attribute Dry or Itchy Skin (IS), we have the following clusters purity.

Table 6. The clusters purity

\begin{tabular}{|l|l|ll|l|}
\hline Cluster Number & Class 1 & Class 2 & Purity \\
\hline 1 & 83 & 46 & & 0.643411 \\
\hline 2 & 43 & 80 & & 0.650407 \\
\hline Overall Purity & & & 80.646909 \\
\hline
\end{tabular}

Further, we present clusters visualization using two dimensional plot. The plot results provide user friendly navigation to understand the cluster obtained. The visualization of the clusters is given in Figure 2.

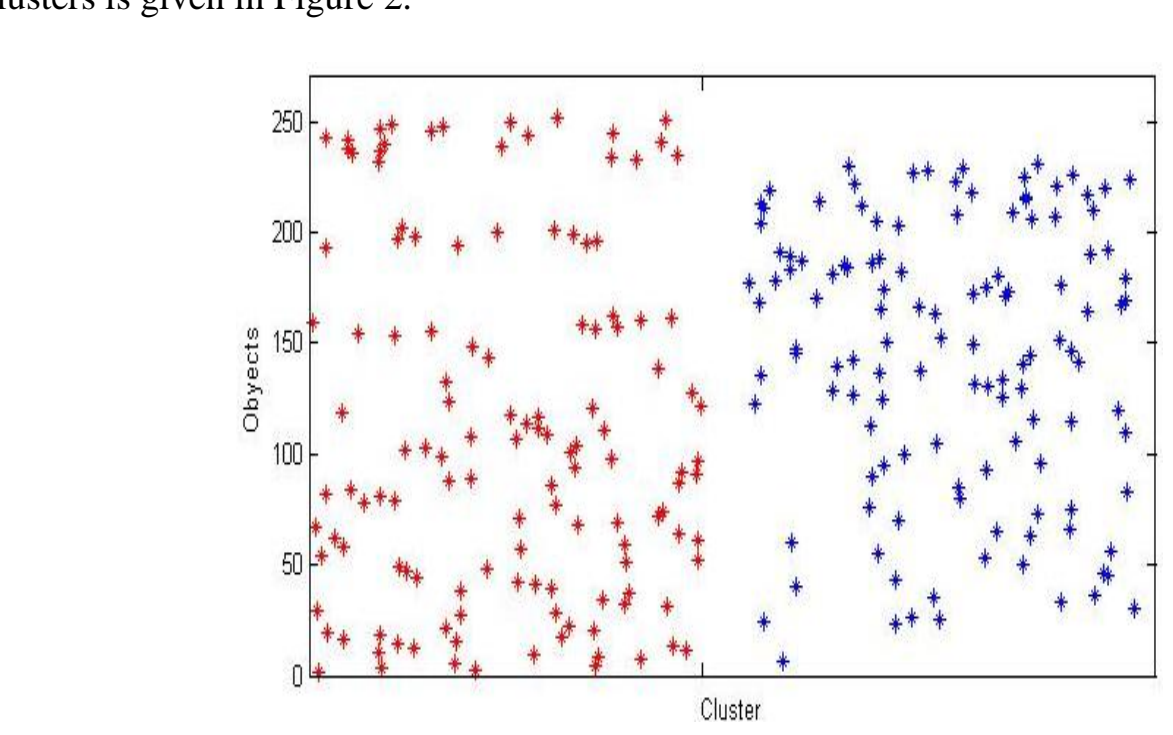

Figure 2. Clusters visualization

\section{Conclusion}

This paper presented an alternative technique for categorical data clustering using Variable Precision Rough Set model. We have shown that the proposed technique able for handling noisy data, as baseline standard rough set-based techniques fail to do. For selecting the clustering attribute, it is based on the maximal of accuracy of approximation using variable 
precision of attributes in the rough set theory. The results of an experimental study through a medical survey diabetic dataset are described and it is shown that our technique provides better performance in selecting the clustering attribute.

\section{Acknowledgements}

The authors thank to Tengku Nurdania Tengku Majani from FSKKP UMP for providing a survey data of patients suspected diabetic. Fundamental Research Grant Scheme (FRGS) from Ministry of Higher Education of Malaysia No. Vote RDU 110104.

\section{References}

[1] P. Berkhin, "Survey of clustering data mining techniques", (2001), http://www.accrue.com/products/rp_cluster_review.pdf, http://citeseet_nl nec.com/berkhino2survey.html.

[2] R. Xu and D. Wunsch, "Survey of Clustering Algorithms", IEEE Transaction on Neural Networks, vol. 16, no. 3, (2005), pp. 645-677.

[3] P. Kumar and B. K. Tripathy, "MMer: an algorthm for clustering heterogeneous data using rough set theory", Int. J. of Rapid Manufacturing, yol. 1, no. 2, (2009), pp. 189207.

[4] Z. Pawlak, "Rough sets", International Journal of Computer and Information Science, vol. 11, (1982), pp. 341-356.

[5] Z. Pawlak, "Rough sets: A theoretical aspect of reasoning about data", Kluwer Academic Publisher, (1991).

[6] Z. Pawlak and A. Skowron, "Rudiments of rough sets", Information Sciences, vol. 177, no. 1, (2007), pp. 327.

[7] L. J. Mazlack, A. He, Y. Zhu and S. Coppock, "A rough set approach in choosing partitioning attributes", In the proceeding of the ISCA 13th, Interhational Conference, CAINE-2000, (2000), pp. 1-6.

[8] D. Parmar, T. Wu and J. Blackhurst, "MMR: An algorithm for clustering categorical data using rough set theory", Data and Knowledge Engineering, vol. 63, (2007), pp. 879-893.

[9] T. Herawan, M. M. Devis and J. H. Abawajy, "Rough set approach for selecting clustering attribute", Knowledge Based Systems, vol. 23, no. 3, (2010), pp. 220-231.

[10] T. Herawan and M.M. Deris, "A framework on rough set-based partitioning attribute selection", In D.S. Huang et al. (Eds): ICIC 2009, Lecture Notes in Artificial Intelligence, vol. 5755, (2009), () Springer-Verlag, pp. 91-100.

[11] W. Ziarko, "Variable precisioh rough set model", Journal of computer and system science, vol. 46, (1991), pp. 39-59.

[12] I. T. R. Yanto, T. Herayan and M. M. Deris, "Data clustering using Variable Precision Rough Set Model", Intelligent Data Analysis, vol. 15, no. 4, (2011), pp. 465-482.

[13] I. T. R. Yanto, P. Vitasari, T. Herawan and M. M. Deris, "Applying Variable Precision Rough Set Model for Clustering Stydent Suffering Study’s Anxiety”, Expert System with Applications, vol. 39, no. 1, (2012), pp. 452-459.

[14] T. Heravan, "On Quasi Discrete Topological Spaces in Information Systems", International Journal of Artificial Life Research, vol. 3, no. 2, (2012), pp. 38-52.

[15] T. Herawan, I. T. R. Yanto and M. M. Deris, "A construction of hierarchical rough set approximations in information systems using dependency of attributes", In N.T. Nguyen et al. (Eds.): Advances in Intelligent Information and Database Systems, Studies in Computational Intelligence, Springer-Verlag, vol. 283, (2010), pp. 3-15.

[16] http://www.diabetes.org.my/article.php?aid=63, (2012). 


\section{Authors}

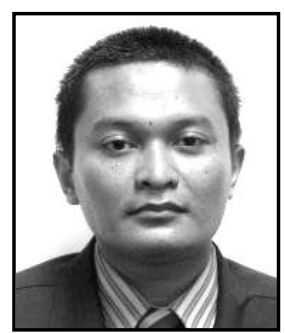

\section{Tutut Herawan}

He received a B.Ed degree in year 2002 and M.Sc degree in year 2006 degree in Mathematics from Universitas Ahmad Dahlan and Universitas Gadjah Mada Yogyakarta Indonesia, respectively. He obtained a PhD in Theoretical Data Mining from Universiti Tun Hussein Onn Malaysia in year 2010. Currently, he is a lecturer with Department of Mathematics Education, Universitas Ahmad Dahlan, Indonesia. He currently supervises four $\mathrm{PhD}$ and had successfully co-supervised two $\mathrm{PhD}$ students and published more than 120 papers in various international journals and conference proceedings. He has appointed as on editorial board member for IJDTA, TELKOMNIKA, IJACAA. IJDCA and IJDIWC. He is also been appointed as a reviewer of several international journals such as Knowledge-Based Systems, Information Sciences, European Journal of Operational Research, Applied Mathematics Letters, and guest editor for several special issues of international journals. He has served as a program committee member and co-organizer for numerous international conferences/Workshops including Soft Computing and Data Engineering (SCDE 2010-2011 at Korea, SCDE 2012 at Brazil), ADMTA 2012 Vietnam, DTA 2011-2012 at Korea, DICTAP 2012 at Thailand, ICDIPC 2012 at Lithuania, DEIS 2012 at Czech Republic, NDT 2012 at Bahrain, ICoCSIM 2012 at Indonesia, ICSDE'2013 at Malaysia, ICSECS 2013 at Malaysia, SCKDD 2013 at Vietnam and many more. His research area includes Knowledge Discovery in Databases, Educational Data Mining, Decision Support in Information System, Rough and Soft Set theory.

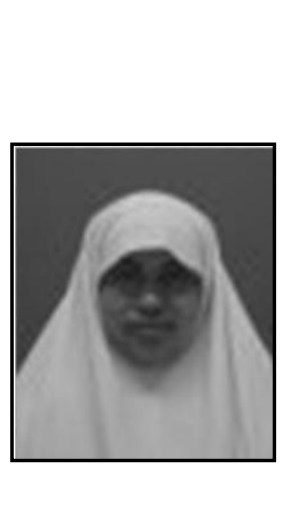

Wan Maseri Wan Mohd

She received a B.Sc. in Computer Science from University of Maimi Florida USA, 1985, a M.Sc. in Computer Science from University of Maimi Florida USA, 1986 and a Ph.D. in Management, University Technology Malaysia in year 2010. Currently she is an associate professor at Faculty of Computer Systems and Software Engineering, Universiti Malaysia Pahang. She is also the director of ICT Business Center, Universiti Malaysia Pahang. She has published more than 30 research papers in journals and conferences. Her research interest includes knowledge management, information retrieval and data mining. 
International Journal of Multimedia and Ubiquitous Engineering Vol.9, No.1 (2014)

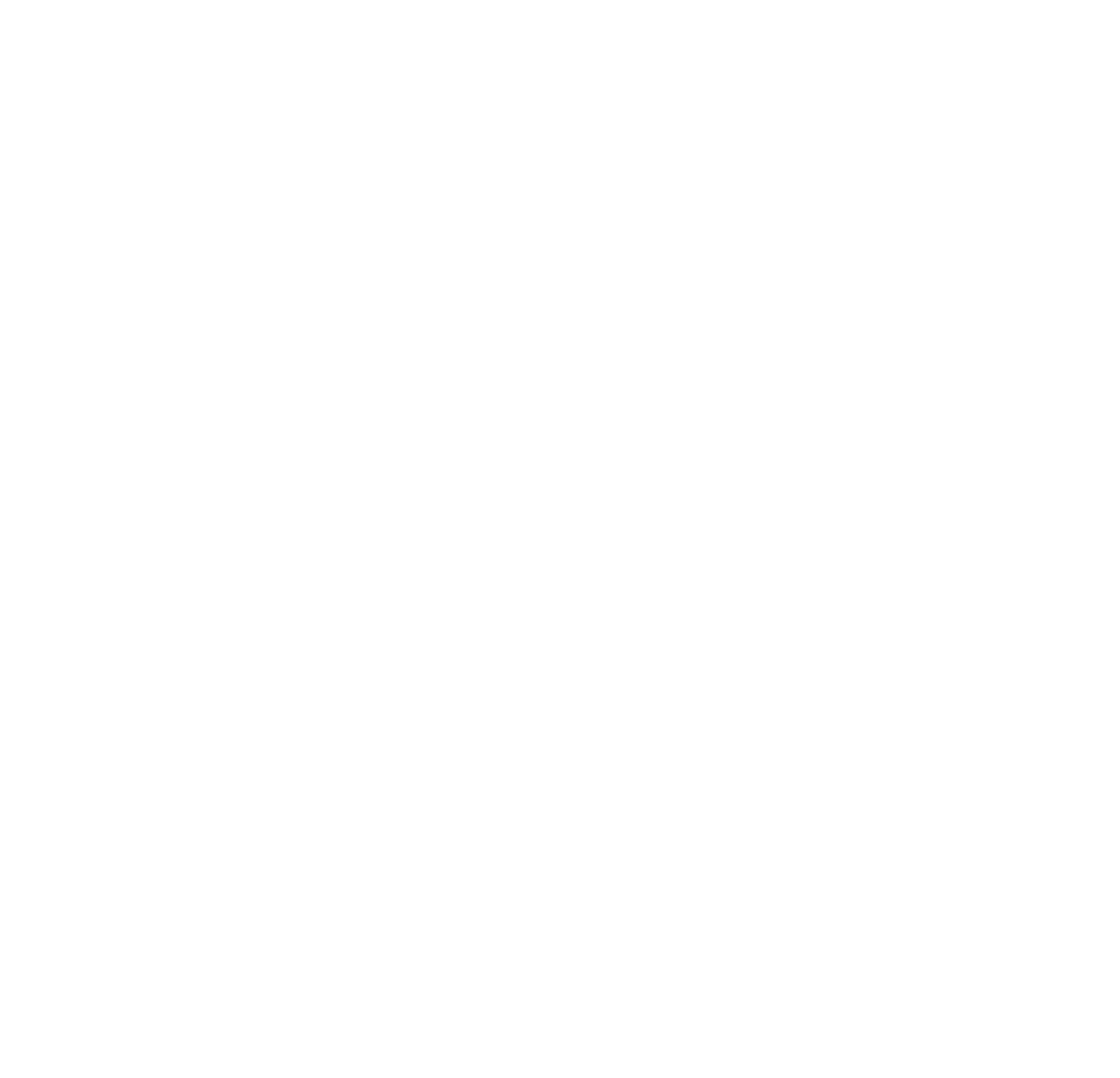

